\title{
Numerical calculation of the rate of crystal nucleation in a Lennard-Jones system at moderate undercooling
}

\author{
Pieter Rein ten Wolde \\ FOM Institute for Atomic and Molecular Physics, Kruislaan 407, 1098 SJ Amsterdam, The Netherlands \\ Maria J. Ruiz-Montero \\ Física Teórica. Facultad de Física. Apdo. de Correos 1065. 41080 Sevilla, Spain \\ Daan Frenkel \\ FOM Institute for Atomic and Molecular Physics, Kruislaan 407, 1098 SJ Amsterdam, The Netherlands
}

(Received 5 January 1996; accepted 22 March 1996)

\begin{abstract}
We report a computer-simulation study of the rate of homogeneous crystal nucleation and the structure of crystal nuclei in a Lennard-Jones system at moderate undercooling. The height of the nucleation barrier has been determined using umbrella sampling, whereas the barrier crossing rate is calculated using molecular dynamics simulation. The simulations clearly show that the barrier crossing is a diffusive process. Nevertheless, the kinetic prefactor in the nucleation rate is found to be some two orders of magnitude larger than predicted by classical nucleation theory. The height of the barrier is in good agreement with the theoretical prediction. Although the Lennard-Jones system has a stable face-centered cubic (fcc) phase below the melting line, the precritical nuclei are found to be mainly body-centered cubic (bcc) ordered. As they grow to their critical size, they become more fcc ordered in the core. However, the critical and postcritical nuclei retain a high degree of bcc ordering in the interface. Furthermore it is found that in the interface the density falls off faster than the structural order parameter, which is in agreement with the predictions of density functional calculations. [P. Harrowell and D. W. Oxtoby, J. Chem. Phys. 80, 1639 (1984)]. (C) 1996 American Institute of Physics. [S0021-9606(96)02224-6]
\end{abstract}

\section{INTRODUCTION}

The formation of a crystalline solid from the bulk liquid phase is generally believed to be an activated process. The height of the free-energy barrier that separates the solid from the (supercooled) liquid is determined by two factors; on the one hand the difference in bulk chemical potential between liquid and solid and, on the other, the interfacial free energy of the crystallite. For small clusters, the interfacial free energy will dominate and hence the free energy will initially increase with cluster size. However, eventually the bulk term will win and sufficiently large crystallites will grow spontaneously.

Although crystal nucleation has been the subject of extensive experimental and theoretical study, ${ }^{1,2}$ our understanding of crystal nucleation at the microscopic level is still limited. One important reason why our knowledge of crystal nucleation is incomplete is precisely because it is an activated process. Experimentally, crystallization rates are typically expressed in units of number of events per second per cubic centimeter. However, the time an incipient crystallite spends at the top of the nucleation barrier is "microscopic" (picoseconds to nanoseconds for atomic, or simple molecular systems). Hence, it is extremely unlikely to find the system exactly at the top of the nucleation barrier. Yet it is exactly the structure and dynamics in this region that plays a crucial role in all theories of crystal nucleation. It is for this reason that computer simulation is a natural tool to study crystal nucleation.

The most straightforward application of molecular dy- namics simulations to crystal nucleation would be to supercool the system and then simply wait for nuclei to appear. However, this approach cannot be used to study nucleation under typical experimental conditions (at $\approx 20 \%$ undercooling with respect to the melting temperature ${ }^{1}$ ). In computer simulations, we are limited to studying relatively small systems (say, of the order of a million particles or less). This means that the volume of the simulation box is less than $10^{-16} \mathrm{~cm}^{3}$. Experimentally observable nucleation rates of the order of one nucleus per $\mathrm{cm}^{3}$ per $\mathrm{s}$, would correspond to the formation of one nucleus per $10^{16} \mathrm{~s}$ in our simulation box. If we take a typical value of $10^{-14} \mathrm{~s}$ for the length of the molecular dynamics time step, then to observe on average one nucleation event, $10^{30}$ time steps would be needed in a simulation of $10^{6}$ particles. As the nucleation barrier decreases as $1 / \Delta T^{2}$, where $\Delta T$ is the degree of supercooling, very large supercoolings (in the order of $40 \%$ ) have to be imposed to observe spontaneous crystal nucleation in a conventional simulation.

However, in the present work, we are interested in crystal nucleation at moderate, i.e., more realistic undercooling. We therefore separate the problem into two parts; (1) the computation of the free-energy barrier for crystal nucleation and (2) the computation of the rate at which this barrier is crossed. For the computation of the free-energy barrier that separates the solid phase from the undercooled liquid, we use the scheme developed by Van Duijneveldt and Frenkel. ${ }^{3}$ The rate at which this barrier is crossed is computed using the Bennett-Chandler scheme to simulate barrier crossing rates. $^{4-7}$ 
As an application, we study crystal nucleation in the Lennard-Jones system. There are two reasons to study this specific system. First of all, there is a considerable body of numerical data available on the phase behavior of this system, while also crystal nucleation under conditions of large supercooling has been studied. The second reason is that the nucleation mechanism in this system has been the subject of debate for several decades. Below, we briefly sketch the background of this debate.

\section{A. Background}

At the end of last century Ostwald ${ }^{8}$ formulated his socalled "step rule," which states that the phase that is formed from the melt need not be the most stable phase, but rather the phase that is closest in free energy to the liquid phase. Stranski and Totomanow ${ }^{9}$ re-examined this rule and argued that the nucleated phase is the phase that has the lowest free-energy barrier of formation, rather than the phase that is globally stable under the conditions prevailing. More recently Alexander and McTague ${ }^{10}$ extended the Landau free energy expansion to freezing transitions that are weakly first order and concluded from general symmetry considerations that, in three dimensions, formation of the body-centeredcubic phase (bcc) phase is uniquely favored for simple fluids. A theoretical study by Klein and Leyvraz ${ }^{11}$ also suggests that a metastable bcc phase can easily be formed from the undercooled liquid. In experiments on rapidly cooled metal melts, which have a stable face-centered-cubic (fcc) phase below the melting line, nucleation of a metastable bcc phase has been observed. ${ }^{12-14}$

However, when the formation of metastable bcc nuclei was investigated on a microscopic scale using computer simulation, the picture that emerged was not fully in agreement with the Alexander-McTague scenario. Although in some studies nucleation of the metastable bcc phase was observed, ${ }^{3,15-17}$ most studies found evidence for the formation of the stable fcc phase. ${ }^{18-23}$ Of particular interest is a simulation study by Swope and Andersen ${ }^{23}$ of a one million particle Lennard-Jones system, which has a stable fcc phase below the melting line. This study showed that although both bcc and fcc crystallites are formed in the early stages of the nucleation process, only the fcc nuclei grow and become postcritical. What should be stressed, however, is that they performed their simulation at a very large degree of undercooling (of more than $40 \%$ with respect to the melting temperature). At such a large degree of undercooling one should expect the free-energy barrier to vanish for essentially all possible crystalline phases. It is therefore not obvious that crystal nucleation at large undercooling will proceed in the same way as close to coexistence.

In the present work we study homogeneous nucleation in the Lennard-Jones system for two different pressures closer to the freezing point, i.e., at $20 \%$ supercooling. The aim of our study was to compute not only the nucleation barrier and the nucleation rate, but also to examine the structure of the precritical, critical, and postcritical nuclei.

\section{B. Summary of results}

Our simulations suggest that the small precritical nuclei have a bcc-like structure rather than the stable fcc structure. However, as the crystallites grow to the critical size, their cores become increasingly fcc ordered. Nevertheless, a high degree of bcc ordering in the interface is retained. This may explain why in earlier simulations on small systems nucleation of a metastable bcc phase was observed, ${ }^{3,15-17}$ while in similar simulations on larger systems the formation of the fcc nuclei was observed. ${ }^{18-23}$ In the smaller systems the critical nuclei will be so small that their structure is almost completely surface dominated, leading to a high degree of bcc ordering.

Our simulations show that, although the density in the core of the critical nuclei is slightly lower than the density in the bulk solid, the structural order parameter reaches a bulk solid value in the core. The interface between the crystal nuclei and the surrounding liquid is diffuse-both the density and the structural order parameter decay smoothly to a liquidlike value. Moreover, our simulations support the prediction from density-functional theory ${ }^{1}$ that the density falls off faster than the structural order parameter.

We compare our numerical results with the predictions of classical nucleation theory for the height of the barrier and the rate of barrier crossing. On the whole, the computed barrier height is in quite good agreement with classical nucleation theory. The present study clearly shows that the barrier-crossing is a diffusive process. This is in agreement with the low Zeldovich factor given by classical nucleation theory. The simulations yield a kinetic prefactor that is two orders of magnitude larger than the one predicted by classical nucleation theory. To our knowledge, the present simulations provide the first "atomistic" calculation of a crystalnucleation rate at moderate undercooling.

The rest of this paper is organized as follows. In Sec. II we describe the numerical techniques to calculate the freeenergy barriers and the nucleation rates. The method of identifying solidlike particles and determining the crystal structure of the nuclei is presented in Sec. III. In Sec. IV we give the computational details of the simulation and in Sec. V we discuss the results. Part of these results were presented in a preliminary report of this study. ${ }^{24}$

\section{NUMERICAL TECHNIQUE}

\section{A. Free-energy barriers}

In order to compute the free-energy barrier that separates the liquid from the crystalline phase, we should first define a "reaction coordinate" that connects the two phases. It is most convenient to choose as the reaction coordinate an, as yet unspecified, order parameter $\Phi$, that is sensitive to the degree of crystallinity in the system. The Gibbs free energy of the system, $G$, is a function of this order parameter, ${ }^{25}$

$$
G(\Phi)=\text { constant }-k_{B} T \ln [P(\Phi)],
$$

where $P(\Phi)$ is the probability per unit interval to find the order parameter around a given value of $\Phi$. Below the freez- 
ing point $P(\Phi)$ is strongly peaked around a finite, solidlike value of $\Phi$, whereas above the freezing point $P(\Phi)$ will be peaked around a low, liquidlike value. At coexistence $P(\Phi)$ is double-peaked and the area under the two peaks should be the same. This expresses the fact that, at coexistence, the system is equally likely to be in the solid or liquid phase.

In the isobaric-isothermal ensemble ( $N P T$-ensemble) the probability $P(\Phi) d \Phi$ that the system has a value between $\Phi$ and $\Phi+d \Phi$ is given by

$P(\Phi)=\frac{\int d V \int d \mathbf{q}^{N} \exp \left\{-\beta\left[U\left(\mathbf{q}^{N}\right)+P V\right]\right\} \delta\left[\Phi-\Phi\left(\mathbf{q}^{N}\right)\right]}{Q_{N P T}}$,

where $\beta \equiv 1 / k_{B} T$ is the reciprocal temperature, $T$ is the temperature, $k_{B}$ is the Boltzmann constant, $N$ is the number of particles, $U\left(\mathbf{q}^{N}\right)$ is the potential energy of the configuration with coordinates $\mathbf{q}^{N}, V$ is the volume, $P$ is the applied pressure, and $Q_{N P T}$ is the configurational part of the partition function. $Q_{N P T}$ is given by

$$
Q_{N P T}=\int d V \int d \mathbf{q}^{N} \exp \left\{-\beta\left[U\left(\mathbf{q}^{N}\right)+P V\right]\right\} .
$$

As $P(\Phi)$ is an equilibrium property of the system it can be obtained both by Monte Carlo (MC) and molecular dynamics (MD) simulations. In order for Eq. (1) to be useful, one should compute $P(\Phi)$ for all values of $\Phi$ between the solid and liquid. In particular, one should obtain an accurate measure of $P(\Phi)$ near the top of the nucleation barrier. But this is precisely the point where $P(\Phi)$ will be very small. As a consequence, Eq. (1) cannot be used to calculate $G(\Phi)$ in a conventional simulation. To circumvent this problem and to obtain good statistics on $P(\Phi)$ for intermediate values of $\Phi$, the umbrella sampling technique of Torrie and Valleau ${ }^{26}$ is used. The basic idea of this scheme is to bias the sampling of configuration space in such a way that configurations with a large free energy will be sampled frequently.

We can bias the sampling of configuration space by adding a fictitious potential to the true potential-energy function of our model system. Clearly, the optimum choice for the biasing potential would be $-G(\Phi)$, because in that case all values of $\Phi$ are sampled with the same probability. But, of course, we do not know $G(\Phi)$ as it is precisely the quantity that we wish to compute. The approach of Van Duijneveldt and Frenkel ${ }^{3}$ and Lynden-Bell et al. ${ }^{27}$ was to construct the biasing potential step-by-step. An initial, local, estimate of $G(\Phi)$ is obtained from an unbiased simulation of the liquid phase (say). This estimate is then extrapolated to higher values of $\Phi$ and used to construct the biasing potential for the next run (at higher values of the order parameter), and so on. The disadvantage of this approach is that if simulations are performed on a large system with a steep free-energy barrier, it becomes difficult to obtain a good estimate for the freeenergy barrier. In the present paper, we therefore use a slightly different approach in that we chose our biasing potential $W(\Phi)$ to be a harmonic function of $\Phi$,

$$
W\left[\Phi\left(\mathbf{q}^{N}\right)\right]=\frac{1}{2} k_{\Phi}\left[\Phi\left(\mathbf{q}^{N}\right)-\Phi_{0}\right]^{2} .
$$

The result of introducing such a potential is that in each run a certain window of values of the order parameter will be sampled. Note that the width and "location" of this window depend on $k_{\Phi}$ and $\Phi_{0}$. The window will be wider if the harmonic constant $k_{\Phi}$ is smaller. By changing the center value of the harmonic potential, $\Phi_{0}$, we can change the crystallinity in our system.

\section{B. Nucleation rates}

With umbrella sampling it is in principle possible to compute the free-energy barrier that separates the liquid from the solid phase, but it does not provide us with any dynamical information. In order to calculate the nucleation rate, we exploit the fact that nucleation is an activated process and that the rate of nucleation can therefore be considered as the product of two terms, namely, (1) the probability to find the system at the top of the free-energy barrier to nucleation and (2) the rate at which this activated state (a "transition state" in the Eyring picture of chemical reactions ${ }^{28}$ ) transforms into a stable crystalline phase. Denoting the transition state separating the liquid from the solid state by $\Phi^{*}$, we consider configurations for which $\Phi<\Phi^{*}$ as liquid and configurations for which $\Phi>\Phi^{*}$ as solid. We now apply standard linear-response theory ${ }^{29}$ to calculate chemical rate constants, to compute the actual transition rate from the liquid to the solid state. ${ }^{5,6}$ This transition rate $k(t)$ is given by ${ }^{5}$

$$
k(t)=\frac{\left\langle\dot{\Phi} \delta\left(\Phi-\Phi^{*}\right) \theta\left[\Phi(t)-\Phi^{*}\right]\right\rangle}{\left\langle\theta\left(\Phi^{*}-\Phi\right)\right\rangle},
$$

where $\theta$ is the Heaviside function. Equation (5) is obtained under the assumption that the actual time scale on which crystallization takes place is very long compared to the time that it takes a critical nucleus to move away from the top of the barrier. It should also be noted that it is somewhat suspect to apply the (equilibrium) linear-response formalism to a system that has been prepared far from equilibrium (namely, in the metastable liquid phase). However, we consider an ensemble of systems, most of which will be in the crystalline state, while a small fraction will be in the metastable liquid. The linear-response theory then tells us how the system relaxes after an initial, weak perturbation has changed the number of systems in the metastable liquid phase. It is in the spirit of the Onsager regression hypothesis ${ }^{29}$ to assume that this relaxation rate is precisely the crystallization rate that we are interested in.

In what follows, we make the assumption that the ratelimiting step in the crystallization rate is the barrier crossing, rather than for instance, the subsequent crystal growth. Therefore, we can identify the crystallization rate with the nucleation rate. 
It is convenient to rewrite Eq. (5) as

$$
\begin{aligned}
k(t) & =\frac{\left\langle\delta\left(\Phi-\Phi^{*}\right)\right\rangle}{\left\langle\theta\left(\Phi^{*}-\Phi\right)\right\rangle} \frac{\left\langle\dot{\Phi} \delta\left(\Phi-\Phi^{*}\right) \theta\left[\Phi(t)-\Phi^{*}\right]\right\rangle}{\left\langle\delta\left(\Phi-\Phi^{*}\right)\right\rangle} \\
& =P_{0}\left(\Phi^{*}\right) R(t) .
\end{aligned}
$$

It is seen that $k(t)$ is the product of two contributions. The first contribution is $P_{0}\left(\Phi^{*}\right)$, which is given by

$$
P_{0}\left(\Phi^{*}\right)=\frac{P\left(\Phi^{*}\right)}{\int_{0}^{\Phi^{*}} d \Phi P(\Phi)}=\frac{\exp [-\beta G(\Phi)]}{\int_{0}^{\Phi^{*}} d \Phi \exp [-\beta G(\Phi)]} .
$$

Noting that if $\Phi<\Phi^{*}$ the system is in the liquid state, it is clear that $P_{0}\left(\Phi^{*}\right)$ is the probability of finding the system at the top of the barrier divided by the probability of finding it in the liquid state. It is an equilibrium quantity and can be measured both by Monte Carlo and by molecular dynamics as indicated above.

The second contribution to $k(t)$ is $R(t)$, which gives the average flux over the top of the barrier, provided that the system was prepared at the top of the barrier. $R(t)$ is a dynamical quantity and can only be measured by molecular dynamics. The basic idea to separate the simulation into a calculation of the barrier height and a dynamic simulation of trajectories starting at the top of the barrier, was formulated by Bennett ${ }^{4}$ and Chandler. ${ }^{5}$ As explained in Ref. 5 the initial rate $k\left(t \rightarrow 0^{+}\right)$corresponds to the transition-state theory approximation for the rate constant,

$$
k_{\mathrm{TST}}=\lim _{t \rightarrow 0^{+}} k(t)=\frac{\left\langle\dot{\Phi} \delta\left(\Phi-\Phi^{*}\right) \theta[\dot{\Phi}]\right\rangle}{\left\langle\theta\left(\Phi^{*}-\Phi\right)\right\rangle} .
$$

Transition-state theory assumes that all trajectories initially heading from the top of the barrier towards the solid state will indeed end up in the solid state and all trajectories heading towards the liquid, will end up in the liquid. This assumption is only correct if no trajectories recross the top of the barrier. In the present case, recrossing turns out to be quite significant and, as a consequence, we will find that $k(t)$ decays to a value that is much smaller than $k_{\mathrm{TST}}$. It is conventional to express the reduction of $k(t)$ due to recrossings in terms of the transmission coefficient $\kappa$, defined as

$$
\kappa=\frac{k(t)}{k_{\mathrm{TST}}}=\frac{R(t)}{R\left(0^{+}\right)} .
$$

As explained in the previous section, we use umbrella sampling to calculate the free-energy barrier and hence, $P\left(\Phi^{*}\right)$. To compute the crossing rate $R(t)$, we make use of the so-called "blue-moon ensemble" technique of Refs. 6 and 7. In this technique, constrained MD simulations are used to generate a sequence of uncorrelated configurations of the system under the constraint $\Phi=\Phi^{*}$ (i.e., at the top of the barrier). We use conventional constraint-MD (Ref. 30) to keep the system at the top of the barrier. However, it should be noted that the quantity that we constrain is a global order parameter that depends on the positions of all the particles in the system. The configurations at the top of the barrier that are generated in the constrained-MD simulations are then used as initial states to compute the time correlation function in $R(t)$. However, as explained in Refs. 6,7, the use of a constrained MD introduces a bias in the sampling of states at the top of the barrier. It is possible to correct for this bias by giving the trajectories starting from the top of the barrier an appropriate weight in the averaging,

$$
R(t)=\frac{\left\langle\dot{\Phi} \theta\left[\Phi(t)-\Phi^{*}\right]|H|^{-1 / 2}\right\rangle_{c}}{\left\langle|H|^{-1 / 2}\right\rangle_{c}} .
$$

The subscript $c$ denotes that we are using a constrained initial state. In the general case of a system with many constraints, $|H|$ is the determinant of a matrix $H$. However, in the present case, there is only one constraint and $H$ reduces to a scalar

$$
H=\sum_{i=1}^{N} m_{i}^{-1}\left(\frac{\partial \Phi}{\partial \mathbf{r}_{i}}\right)^{2}
$$

The weighting factors in the ratio in Eq. (10) would cancel if the reaction coordinate $\Phi$ were a linear function of the cartesian coordinates. However, in the present case, $\Phi$ is a nonlinear function of all coordinates and its influence cannot be ignored. More computational details will be discussed in Ref. 31.

\section{Order parameters}

Both for the calculation of the nucleation barrier and for the computation of the crossing rate, we need to define a "reaction" coordinate that measures the degree of crystallinity of the system as it moves from the liquid to the solid phase. We have to choose as our reaction coordinate an order parameter that is only sensitive to the overall degree of crystallinity of the system, but fairly insensitive to the differences between the various possible crystal structures. This requirement is important because otherwise we would force the system to go towards a specific crystal structure. A second requirement is that the order parameter should be insensitive to the orientation of the crystal in space. Van Duijneveldt and Frenkel ${ }^{3}$ have shown that a particular set of bondorder parameters introduced by Steinhardt et al. ${ }^{32}$ are particularly suited to act as the reaction coordinate. These order parameters are sensitive to the degree of spatial orientational correlation of the vectors that join neighboring particles. In a liquid where there is only local orientational order, these correlations decay rapidly and, as a consequence, all bond-order parameters are small (zero in the thermodynamic limit). In a crystal, the orientation of vectors joining neighboring atoms are correlated throughout the solid and hence the bond-order parameter is large [of $\mathscr{Q}(1)]$.

In Appendix A we briefly summarize the definition of the bond-order parameters used in our simulations. In Table I values for several of these order parameters are given for simple cluster geometries. As can be seen from Table I, $Q_{6}$ has the desirable feature that it vanishes in the bulk liquid phase, while it is large [O(1)] for the simple crystal lattices of interest. We therefore use $Q_{6}$ as the crystalline order parameter. The reaction coordinate from isotropic fluid to crystal then corresponds to a path of increasing $Q_{6}$. By increas- 
TABLE I. Bond orientational order parameters for a number of simple cluster geometries. fcc, face-centered-cubic structure; hcp, hexagonal closepacked structure; bcc, body-centered-cubic structure, and sc, simple cubic structure.

\begin{tabular}{llllr}
\hline \hline & $Q_{4}$ & $Q_{6}$ & $\widehat{W}_{4}$ & $\widehat{W}_{6}$ \\
\hline fcc & 0.191 & 0.575 & -0.159 & -0.013 \\
hcp & 0.097 & 0.485 & 0.134 & -0.012 \\
bcc & 0.036 & 0.511 & 0.159 & 0.013 \\
sc & 0.764 & 0.354 & 0.159 & 0.013 \\
Icosahedral & 0 & 0.663 & 0 & -0.170 \\
(liquid) & 0 & 0 & 0 & 0 \\
\hline \hline
\end{tabular}

ing $Q_{6}$ from the liquid we do not favor a specific crystalline structure. Rather, the system is allowed to select its "own" specific reaction path from the fluid to one of the crystal structures listed in Table I. The other order parameters listed in Table I were used to analyze the configurations and distinguish between different crystal structures.

\section{STRUCTURE ANALYSIS}

Although the concept of a crystal nucleus is intuitively clear, it is not easy to give an unambiguous numerical criterion that will identify atoms as either solid- or liquidlike. In fact, a great variety of criteria to identify solidlike clusters in the liquid have been proposed. Here, we briefly review those criteria that are based on the structure (rather than the dynamics) of crystalline nuclei. In the earliest simulation studies of nucleation in a Lennard-Jones system, Mandell et al. ${ }^{15}$ used the "local" structure function in order to identify crystalline nuclei. The main disadvantage of the method is that it does not have high spatial resolution and, more seriously, can be rather sensitive to the orientation of the crystal nuclei. The structure analysis used by Honeycutt and Andersen ${ }^{33}$ is based on the observation ${ }^{34}$ that there are many nearly collinear triplets of neighboring particles in the Lennard-Jones solid, whereas there are comparatively few such triplets in the liquid. The criterion used by Honeycutt and Andersen for deciding whether a given atom was solidlike, was that the atom must have at least five distinct pairs of its nearest neighbors with which it forms a triplet whose angle is greater than a specified cutoff angle near $180^{\circ}$. However, they observed that the size of the critical nucleus strongly depends on the cutoff angle used. Yang et al. ${ }^{35}$ adopted a criterion that is based on the observation that crystalline solids, unlike liquids, can be constructed by periodically repeating a unit cell. In Ref. 35 solidlike regions are identified by searching for such periodically repeating units.

A more widely used technique for studying both crystalline and amorphous structures is the Voronoi-analysis of the topology of the environment of a given particle.,16,17,19,21,23 The Voronoi polyhedron associated with a given particle is defined as the set of all points of space that are closer to that particle than to any of the others. In a perfect crystal, the Voronoi polyhedron reduces to the Wigner-Seitz cell. It is customary to define the signature of a Voronoi polyhedron as a set of integers $\left(n_{3}, n_{4}, n_{5}, \ldots\right)$, where $n_{l}$ is the number of $l$-sided faces of the polyhedron. For example, the Voronoi polyhedron of a perfect fcc structure, the rhombic dodecahedron (that has twelve lozenge-shaped faces), is denoted by $(0$ $1200 \ldots)$, while the Voronoi polyhedron of a particle in a body-centered-cubic (bcc) structure, is denoted by (0 60080 ...) (six squares, eight hexagons).

In practice, the Voronoi signatures of the particles in a crystal will be modified by the thermal vibrations. For instance, the characteristic Voronoi polyhedron of the fcc lattice, the rhombic dodecahedron, will be removed by the $t i$ niest thermal motion. Of the 14 vertices of the rhombic dodecahedron there are six where four faces meet. Any thermal motion will make these fourfold vertices break up into sets of threefold vertices connected by short edges. The result is that a variety of polyhedra such as (0364), (0365), (0446), (0447) occur in a thermally equilibrated fcc crystal. Such tiny displacements of particles do not affect the signature of the bcc Voronoi polyhedron, because it has only threefold vertices. This is why it is often said that the bcc Voronoi polyhedron is stable against thermal distortions. However, although this may be true for cold bcc crystals, we find that a bcc crystal close to melting has many other Voronoi signatures in addition to the characteristic (06080). Hence, Voronoi signatures can only be used in a statistical sense to identify solidlike particles.

\section{A. Identification of crystalline clusters}

In the previous section, we described how we compute the degree of crystallinity of the system using global bondorder parameters. ${ }^{3}$ We have extended this technique to identify individual solidlike particles and hence solid clusters. The advantage of the scheme is that it is rather insensitive to the crystal structure of the cluster.

To identify solidlike particles, we make use of the local orientational order parameter $\bar{q}_{l m}(i)$ as defined in Eq. (A1). From the $\bar{q}_{l m}(i)$ we can construct local invariants,

$$
q_{l}(i) \equiv\left[\frac{4 \pi}{2 l+1} \sum_{m=-l}^{l}\left|\bar{q}_{l m}(i)\right|^{2}\right]^{1 / 2}
$$

and

$$
\hat{w}_{l}(i) \equiv w_{l}(i) /\left[\sum_{m=-l}^{l}\left|\bar{q}_{l m}(i)\right|^{2}\right]^{3 / 2},
$$

with $w_{l}(i)$ given by

$$
\begin{aligned}
w_{l}(i) \equiv & \sum_{\substack{m_{1}, m_{2}, m_{3} \\
m_{1}+m_{2}+m_{3}=0}}\left(\begin{array}{ccc}
l & l & l \\
m_{1} & m_{2} & m_{3}
\end{array}\right) \\
& \times \bar{q}_{l m_{1}}(i) \bar{q}_{l m_{2}}(i) \bar{q}_{l m_{3}}(i) .
\end{aligned}
$$

These local order parameters are measures for the local order around particle $i$. However, the local order is large not only in the solid, but also in the liquid. Hence, both in the liquid and in the solid the local order parameters $q_{l}(i)$ are nonzero, see Fig. 1. The reason that nevertheless a global order parameter, such as $Q_{6}$, vanishes in the liquid, is that all $\bar{q}_{6 m}(i)$ add up incoherently. In the solid, the $\bar{q}_{6 m}(i)$ add up coherently and, as a consequence, the global order param- 

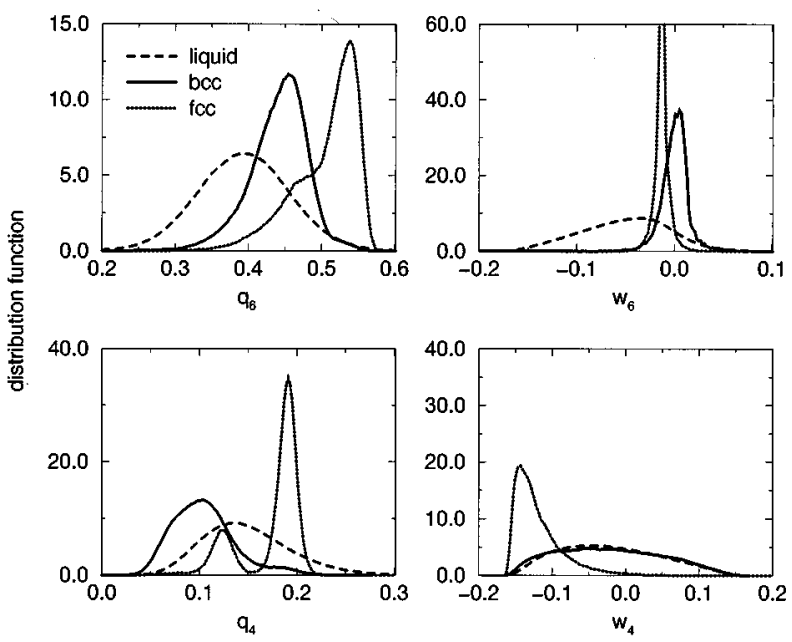

FIG. 1. Probability distribution functions of the local order parameters, as defined in Eqs. (12) and (13), in a Lennard-Jones system for a thermally equilibrated liquid, bcc and fcc structure at $20 \%$ undercooling $(P=5.68$, $T=0.92$ ). The distribution functions are based on averages over 50 independent atomic configurations.

eters are nonzero. It is this coherence of local bond-order parameters that we use to identify solidlike particles.

To every particle $i$ we attribute a normalized $(2 \times 6+1)$-dimensional complex vector $\mathbf{q}_{6}(i)$, with components

$$
\tilde{q}_{6 m}(i) \equiv \frac{\bar{q}_{6 m}(i)}{\left[\sum_{m=-6}^{6}\left|\bar{q}_{6 m}(i)\right|^{2}\right]^{1 / 2}} .
$$

We can now define a dot product of the vectors $\mathbf{q}_{6}$ of neighboring particles $i$ and $j$,

$$
\mathbf{q}_{6}(i) \cdot \mathbf{q}_{6}(j) \equiv \sum_{m=-6}^{6} \widetilde{q}_{6 m}(i) \widetilde{q}_{6 m}(j)^{*} .
$$

By construction, $\mathbf{q}_{6}(i) \cdot \mathbf{q}_{6}(i)=1$.

We now consider particles $i$ and $j$ to be "connected" if the dot-product $\mathbf{q}_{6}(i) \cdot \mathbf{q}_{6}(j)$ exceeds a certain threshold, in our case 0.5 . It is clear that in the solid almost all $\mathbf{q}_{6}(i)$ are in phase with one another and add up coherently to produce a nonzero $\bar{Q}_{6 m}$. Using this criterion all particles in the solid will turn out to be connected with one another. However, to identify a particle as "solidlike," it is not enough that its bond-order is in phase with only one of its neighbors. After all, even in the liquid it will frequently happen that the bondorder of neighboring particles is in phase and hence the two particles are considered "connected." We therefore only identify a particle as solidlike if the number of connections with its neighboring particles exceeds a threshold value. To illustrate this technique, Fig. 2 shows the histograms of the number of connections per particle for the liquid, the bcc structure and the fcc structure of the Lennard-Jones system, all equilibrated at the fcc-liquid coexistence point. As is to be expected, the average number of connections per particle in the liquid is less than in either solid. More importantly, the

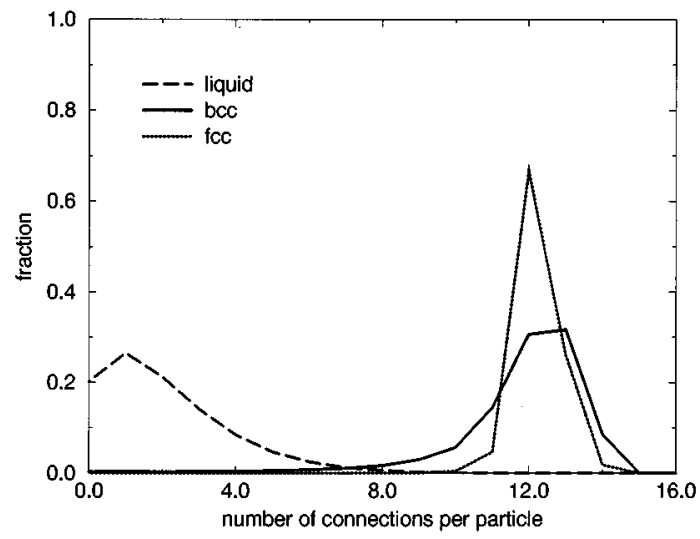

FIG. 2. Distributions of the number of connections per particle in a Lennard-Jones system for a thermally equilibrated liquid, bcc and fcc structure at coexistence $(P=5.68, T=1.15)$. The distributions are based on averages over 50 independent atomic configurations.

histogram for the liquid phase exhibits very little overlap with the histograms of the two solid phases. We find that, with a threshold value of seven connections per particle, more than $99 \%$ of the particles in a fcc structure are identified as being solidlike. Even for the bcc structure, which is rather open and disordered, this method identifies more than 97\% of the particles as solidlike. In contrast, for the liquid less than $1 \%$ of the particles were identified as being solidlike. Thus this analysis method gives an unambiguous, local criterion to identify solidlike particles. Once we have identified the individual solidlike particles, we can perform standard cluster analysis to recognize crystallites. We apply the criterion that any two solidlike particles that are neighbors belong to the same solid cluster.

\section{B. Crystal-structure determination}

As discussed above, the typical Voronoi polyhedra of the different crystal structures will be distorted by thermal vibrations of the particles around their lattice positions. As a consequence, a given structure will be characterized by a distribution of signatures, rather than a single one. In fact, each crystal structure has its own unique distribution of Voronoi signatures. Similarly, every structure has its own unique distribution of local bond-order parameters. We can use either distribution as a "fingerprint" that enables us to identify the crystal structure of crystalline nuclei.

To see how this method of analysis works, consider, for instance, the Voronoi histogram of a solid cluster. We represent this histogram as an $n$-dimensional unit vector $\hat{\mathbf{v}}$, where the number of components $(n)$ corresponds to the number of "bins" of the histogram. We then decompose the vector $\hat{\mathbf{v}}$ corresponding to the cluster in a linear combination of the corresponding vectors for the equilibrated liquid, bcc and fcc structures. That is, we minimize

$$
\Delta^{2}=\left[\hat{\mathbf{v}}_{\mathrm{cl}}-\left(f_{\mathrm{liq}} \hat{\mathbf{v}}_{\mathrm{liq}}+f_{\mathrm{bcc}} \hat{\mathbf{v}}_{\mathrm{bcc}}+f_{\mathrm{fcc}} \hat{\mathbf{v}}_{\mathrm{fcc}}\right)\right]^{2},
$$


where $\hat{\mathbf{v}}_{\mathrm{cl}}, \hat{\mathbf{v}}_{\text {liq }}, \hat{\mathbf{v}}_{\mathrm{bcc}}$ and $\hat{\mathbf{v}}_{\mathrm{fcc}}$ are the vectors associated with the histograms of the cluster, the liquid, the bec structure, and the fcc structure, respectively. Clearly, the coefficients $f_{\text {liq }}, f_{\text {bcc }}$ and $f_{\text {fcc }}$ are indicative of the type of crystal structure of the cluster. The value of $\Delta^{2}$ is an indication of the quality of the fit. For instance, if we were to apply our analysis to an equilibrated fcc crystal, we would find $f_{\mathrm{fcc}}=1$, $f_{\text {bcc }}=0, f_{\text {liq }}=0$, and $\Delta=0$.

Analogously, we can interpret the histogram of the probability distribution function of the local bond-order parameters as a multidimensional vector. Figure 1 shows the probability distribution functions of the most interesting orientational order parameters for the liquid, bcc and fcc structures. The important thing to note is that, although the distributions of the local order parameters are quite broad, in particular in the liquid phase, there is still a considerable difference between the distributions that correspond to different phases. For instance, the distribution of $\hat{w}_{6}(i)$ is strongly peaked in either solid phase, but not in the liquid. The distribution of $q_{4}(i)$ has a characteristic double-peaked structure in the fcc phase, but not in the bcc or liquid phases. We found that the probability distribution of $\hat{w}_{4}(i)$ of the bcc phase is almost identical to that of the liquid. It could still be used to distinguish fcc structures from liquid or bcc. However, we found that the information contained in the $\hat{w}_{4}$-distribution function did not add to the information obtained by using the $q_{4}, q_{6}$, and $\hat{w}_{6}$ distributions. It is only the latter distributions that we have used in our structure analysis. To be more precise, we first concatenate the distribution functions of $q_{6}(i), q_{4}(i)$, and $\hat{w}_{6}(i)$ for each structure to form a single, unique distribution function. With the histogram of this distribution function we then associate a (normalized) vector. As with the Voronoi histograms, we can then decompose the order-parameter histogram of our solid cluster in the components corresponding to pure fcc, bcc, and liquid.

When comparing the structure analysis based on Voronoi histograms with the local bond-order parameter method, we found that the Voronoi method was not very robust; a slight disordering of a bcc crystal led to a strong change in the Voronoi histogram [for instance, the characteristic (0608) signature is almost completely destroyed] and the Voronoi signatures of the disordered bcc and fcc structures end up looking quite similar. For this reason we have only used the more sensitive bond-order histogram method in our structure analysis.

\section{SIMULATIONS}

All simulations were performed in the isobaricisothermal (constantNPT) ensemble. Both Monte Carlo simulations and molecular dynamics simulations were performed. The advantage of MD is that it facilitates equilibration through collective particle motions. Moreover, MD is essential to study the kinetics of crystal nucleation. The advantage of Monte Carlo simulations is that it is particularly suited for umbrella sampling. In what follows, we use re- duced units, such that the Lennard-Jones well depth $\epsilon$ is the unit of energy, while the Lennard-Jones diameter $\sigma$ is the unit of length.

In the Monte Carlo simulations each trial move consisted either of an attempted displacement of a particle or a trial volume change. The choice between trial volume moves and trial particle moves was made at random, with $92 \%$ probability for the latter. The acceptance ratio of the particle moves was maintained at $25 \%$, while that of the volume moves was kept at 50\%. For more details of the Monte Carlo scheme, see Refs. 3,36. In order to keep the pressure and temperature constant in our molecular dynamics simulations, we applied the extended system method proposed by Nosé and Andersen. ${ }^{37}$ The equations of motion were integrated by a predictor-corrector version of the velocity Verlet algorithm ${ }^{30}$ and the time step used in the molecular dynamics simulations was in the range $0.005-0.01 \tau$, where $\tau$ is the unit of time. This was adequate for energy conserving dynamics.

The cutoff radius for intermolecular interactions was chosen such that $r_{c}=2.5$. For the calculation of bond-order parameters, the cutoff distance for nearest-neighbor "bonds" was chosen at $r_{q}=1.5$, which corresponds approximately to the first minimum of $g(r)$ in a fcc crystal at coexistence (in the Monte Carlo simulations the cutoff radii scale with the linear dimensions of the simulation box, but this is a small effect). To minimize the anisotropy in the system due to the periodic boundary conditions, we used a truncated octahedral simulation box. ${ }^{38}$ To speed up the simulation, we used a Verlet neighbor list to calculate energies and forces and a linked list ${ }^{30}$ to update the neighbor list. In Appendix B we describe how we combined the linked list method with truncated octahedral boundary conditions.

All simulations were started from a liquid configuration, obtained by melting a crystal. The first run in a series of umbrella samplings was performed without any weighting function. By changing the biasing potential, the next simulation was performed in an adjacent $Q_{6}$ interval. In this way we could slowly increase the crystallinity in the system and cross the free-energy barrier that separates the liquid phase from the solid phase. Once we had crossed the top of the barrier, we checked whether the path was reversible by lowering $Q_{6}$. We observed no significant hysteresis at the top of the barrier, although very long simulations were required to equilibrate the system.

As the equilibration time and the order-parameter fluctuations are much larger at the top of the barrier than on either side of it, we tuned the biasing potential in such a way that, at the top of the barrier, only narrow windows in $Q_{6}$ were sampled. A typical simulation in a given window consisted of an equilibration period of 10000-50000 cycles (MC)/time steps (MD), followed by a production run of 25 000-75000 cycles/time steps.

The individual probability distribution functions $P\left(Q_{6}\right)$ obtained in different runs were fitted simultaneously to a polynomial. ${ }^{3}$ We used a polynomial fit rather than the selfconsistent procedure of Ferrenberg and Swendsen, ${ }^{39}$ because a good polynomial fit can be obtained even when the adjacent histograms do not overlap or overlap only slightly. The 
reason is that even a very narrow histogram yields an estimate of the local derivative of the free energy. From this local information, the global free-energy barrier can then be reconstructed using a polynomial fit.

Having determined the free energy barrier, we used constrained MD to generate a sequence of configurations at $Q_{6}^{*}$, the position of the top of the barrier. The duration of this MD simulation was of $100 \tau$ (25 000 time steps) and from this run we kept 50 independent configurations separated by $2 \tau$ (500 time steps) to be used as initial states for the computation of the barrier crossing flux $R(t)$, as given by Eq. (10). At the beginning of the unconstrained MD runs to compute $R(t)$, all particles were given a velocity drawn from a Maxwell-Boltzmann distribution. The duration of these runs was $5 \tau$, which appeared long enough for the system to reach a stationary state. In order to improve the statistics, we assigned different initial velocities to the same configurations, and we also made use of the time reversal property,

$$
R(t)=-\frac{\left\langle\dot{Q}_{6} \theta\left[Q_{6}(-t)-Q_{6}^{*}\right]|H|^{-1 / 2}\right\rangle}{\left\langle|H|^{-1 / 2}\right\rangle}=-R(-t) .
$$

This means that the flux was computed by averaging over the trajectories obtained propagating forwards and backwards our set of initial configurations obtained from a constrained run at the top of the barrier. The results that we present here for the rate were averaged over 200 trajectories.

\section{RESULTS AND DISCUSSION}

We studied the formation of a critical nucleus and the rate of nucleation for a Lennard-Jones system at $20 \%$ undercooling with respect to the melting temperature. Although this degree of supercooling is appreciably less than what is used in "brute force" simulations of crystal nucleation, it is still large compared to the degree of supercooling that can be reached experimentally for simple liquids such as argon. In our choice of this particular degree of supercooling we tried to strike a compromise between making the supercooling as small as possible and, at the same time, keeping the critical nucleus much smaller than the system size. As we studied a system of $\mathscr{Q}\left(10^{4}\right)$ particles, we tried to ensure that the supercooling was strong enough to make the critical nucleus at least one order of magnitude smaller. A rough estimate, based on classical nucleation theory, suggests that the size of the critical nucleus is about 100 particles for $20 \%$ undercooling. However, several studies indicate that, although the core of the nucleus might be quite small, the interface between the liquid and the solid is rather diffuse, ${ }^{15,33,40,41}$ so in practice the number of solidlike particles may be appreciably larger. After testing the method on a small system, we performed all production runs on a system of 10648 particles.

We performed the simulations at two different reduced pressures, $P=0.67$ and $P=5.68$. We used the data of Hansen and Verlet ${ }^{42}$ to estimate the location of the melting points (see Table II). Figure 3 shows the free-energy barriers computed for these two pressures. Let us first describe qualitatively what happens as the system crosses the barrier. Ini-
TABLE II. Transition data for the Lennard-Jones system at the reduced pressures $P=0.67$ and $P=5.68$. From Hansen and Verlet (Ref. 42).

\begin{tabular}{cccc}
\hline \hline$P$ & $T$ & $\rho_{\text {liquid }}$ & $\rho_{\text {crystal }}$ \\
\hline 0.67 & 0.75 & 0.875 & 0.973 \\
5.68 & 1.15 & 0.936 & 1.024 \\
\hline \hline
\end{tabular}

tially, the system is in the metastable liquid phase. Due to spontaneous fluctuations, some small solidlike clusters are present in the liquid. We find that the solidlike clusters rarely comprise more than 16 particles. When $Q_{6}$ is increased from the liquid, both the number and size of these solidlike clusters in the liquid increase. The reason why there are, initially, several small solidlike clusters is that is is entropically favorable for the system to distribute a given amount of crystallinity over several clusters. For a given overall degree of crystallinity, there is a competition between translational entropy, favoring the formation of many small clusters, and surface free energy, which favors the formation of a single large crystallite. When the top of the barrier is approached, the surface free energy dominates and the small solidlike clusters merge. Indeed, at the top of the barrier only one cluster, the critical nucleus, is observed, apart from a number of small solidlike fluctuations that are always present in the liquid. This implies that the Gibbs free energy of the system at the top of the barrier corresponds to the Gibbs free energy of the critical nucleus, the nucleation barrier.

In the following we first discuss the structure of the nuclei as a function of our "reaction coordinate." Next, we consider the structure of the critical nucleus in more detail by examining the radial profiles for the density and our structural order parameters. We will only present the results of the structure analysis for the system at $P=5.68$, as the ones for $P=0.67$ are qualitatively similar. Finally, we discuss the transition rate and make a comparison with classical nucleation theory.

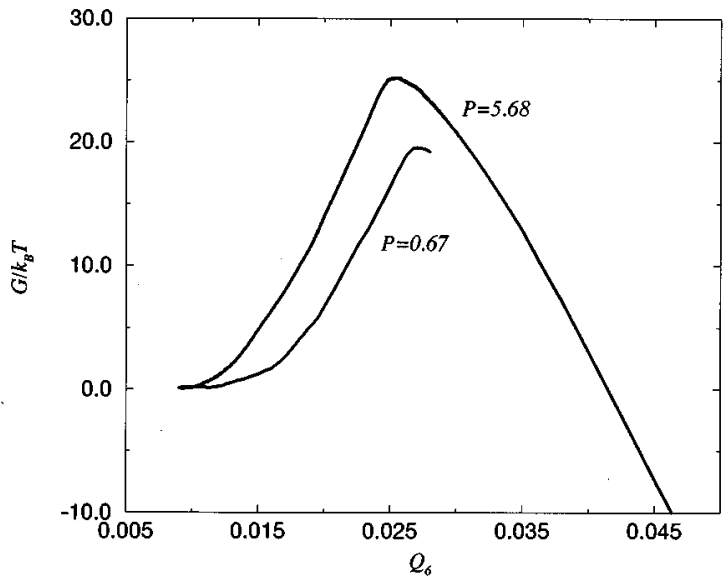

FIG. 3. The Gibbs free energy of a Lennard-Jones system as a function of crystallinity $\left(Q_{6}\right)$ at $20 \%$ undercooling for two different pressures, i.e., $P=5.68(T=0.92)$ and $P=0.67(T=0.6)$. The Gibbs free-energy barriers are approximately $25.1 k_{B} T$ at $P=5.68$ and $19.4 k_{B} T$ at $P=0.67$. 


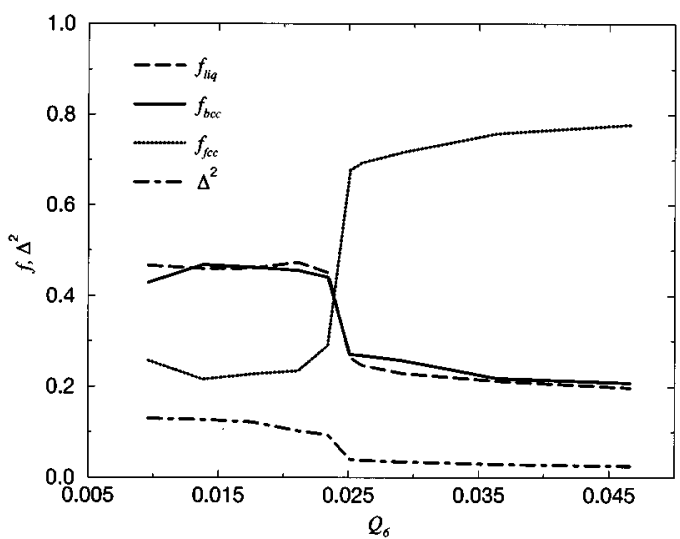

FIG. 4. Structural composition of the largest cluster in a Lennard-Jones system, indicated by $f_{\text {liq }}, f_{\text {bcc }}, f_{\text {fcc }}$, and $\Delta^{2}$, as a function of $Q_{6}$ (the reaction coordinate) at $20 \%$ undercooling $(P=5.68, T=0.92)$. This figure is based on averages over 50 independent atomic configurations.

\section{A. Crystallite structure}

As mentioned in the previous section, only small crystallites are observed on the liquid side of the barrier. The size of the largest crystallites ranges from 16 particles in the metastable liquid to 26 particles as the top of the barrier is approached. Previous theoretical, ${ }^{43}$ experimental, ${ }^{44}$ and computer simulation studies ${ }^{44-46}$ indicate that for small clusters of Lennard-Jones atoms in vacuo the icosahedral structure is more stable than any of the crystalline structures. Besides, it has been suggested ${ }^{32}$ that long-ranged icosahedral order would be favored in strongly supercooled liquids. When we applied a conventional Voronoi analysis to our system in the liquid state, we could identify on average $1 \%$ of the atoms as being icosahedrally surrounded. However, the larger crystallites that were present in the liquid never contained any atom with the characteristic ( $\left(\begin{array}{llll}0 & 0 & 12 & 0\end{array}\right)$ signature of an icosahedron. Also an examination of the local bond order parameter $\widehat{w}_{6}$, which is most sensitive to icosahedral order (see Table I), supported the conclusion that the largest crystallites do not contain icosahedrally ordered atoms. In fact, the bondorder analysis indicates that the larger solidlike clusters in the metastable liquid have appreciable bcc character, whereas at the top of the barrier and beyond, they are predominantly fcc-like. To make this analysis more quantitative, we determined $f_{\text {liq }}, f_{\text {bcc }}$ and $f_{\text {fcc }}$ as defined in Eq. (17) for the largest cluster in the system.

Figure 4 shows the structural "composition" of the largest cluster in the system, as a function of the "reaction coordinate," $Q_{6}$. The figure shows that the precritical nuclei are predominantly bcc- and liquidlike. However, near the top of the barrier, at $Q_{6}=0.025$, there is a clear change in the nature of the solid nuclei from bcc- and liquidlike to mainly fcc-like. The fact that the precritical nuclei are rather liquidlike is not surprising as they are quite small and almost all interface. The important point to note is that these nuclei have clearly more bcc than fcc character. This suggests that, at least for small crystallites, we find the behavior predicted by Landau theory. ${ }^{10}$ Yet, as the critical and postcritical clus-

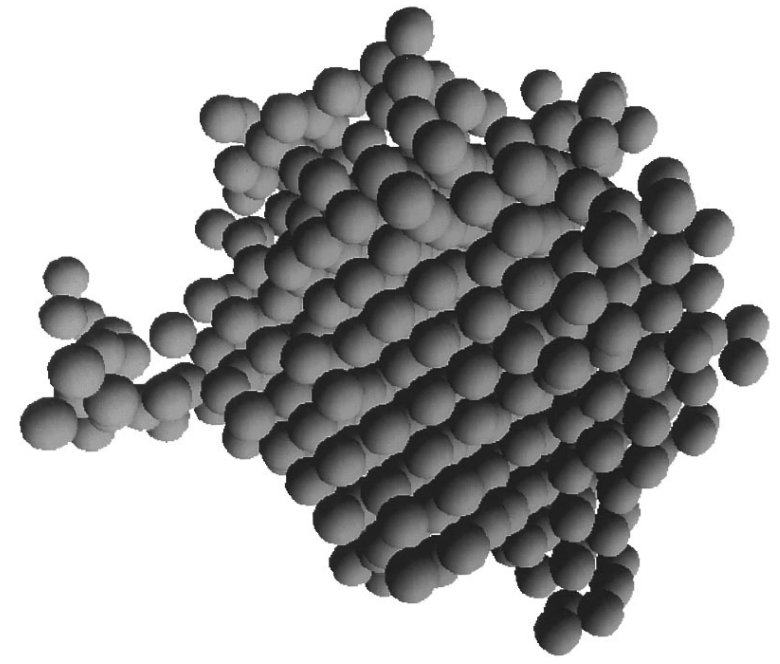

FIG. 5. Snapshot of the critical nucleus at $20 \%$ undercooling $(P=5.68$, $T=0.92)$ in a Lennard-Jones system.

ters are predominantly fcc-like, the present results are also compatible with the findings of Swope and Andersen, ${ }^{23}$ who observed that nucleation proceeded through fcc crystallites. In fact, the nucleation process as observed in the present simulations might be interpreted as a manifestation of the Ostwald step rule. ${ }^{8}$ First, a metastable, bcc, phase is nucleated, which is then transformed into a more stable, fcc, phase. What is remarkable is that we find that the transformation from bcc to fcc takes place before the critical nucleus is reached.

\section{B. Critical nucleus}

Visual inspection of the critical and postcritical nuclei showed that the nuclei at this moderate degree of undercooling are fairly compact, more or less spherical objects (see Fig. 5). This finding appears to be in contrast to what is found in simulations of crystal nuclei at large supercooling ${ }^{33,35}$ where ramified structures were observed. Although we find the critical nucleus to be fairly spherical, rudimentary facets can be distinguished. Facetting of crystal nuclei was also observed by Báez and Clancy, ${ }^{47}$ who studied the growth and dissolution of critical fcc nuclei implanted in a liquid at $26 \%$ undercooling. Báez and Clancy found that during the earliest stages of growth the nuclei are distinctly octahedral, with facets corresponding to the (111) planes of the fcc crystal.

In order to quantify the degree of nonsphericity of the critical nucleus, we expand the mass distribution of the crystallite in rank four spherical harmonics $\left(Y_{4 m}\right)$ and constructed quadratic invariants, denoted by $S_{4}(\mathrm{cl})$. For a spherical cluster $S_{4}(\mathrm{cl})$ is, of course, zero. But for an octahedral cluster it has a value of 0.11 . We find that, both for the critical and postcritical nuclei, $S_{4}(\mathrm{cl})$ is much smaller than is compatible with an octahedral shape. Hence the critical and postcritical nuclei in our simulations are indeed quite spherical, which supports the assumption of classical nucleation theory. However, this finding seems hard to reconcile with 


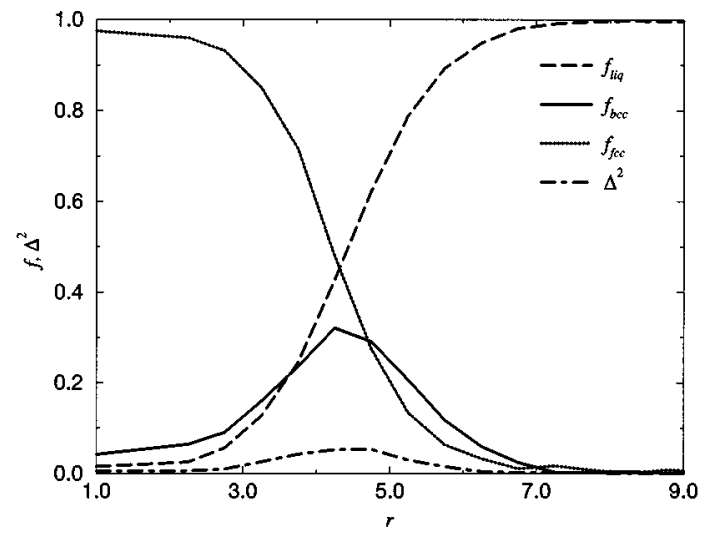

FIG. 6. Structure of the critical nucleus, indicated by $f_{\text {liq }}, f_{\text {bcc }}, f_{\text {fcc }}$, and $\Delta^{2}$, as a function of $r$, the distance to its center-of-mass, at $20 \%$ undercooling $(P=5.68, T=0.92)$ in a Lennard-Jones system. This figure is based on averages over 50 independent atomic configurations.

the strong faceting of crystal nuclei that was observed by Báez and Clancy. ${ }^{47}$ It should be recalled that Broughton and Gilmer, who have computed the interfacial free energy of a Lennard-Jones system for three different orientations of the fcc crystal-liquid interface, ${ }^{48}$ found the surface free energies for the (111), (100), and (110) faces to be equal to within the statistical error. If the interfacial free energy is indeed completely isotropic, one should expect to see a spherical crystal nucleus. Slight anisotropies in the interfacial free energy might lead to fairly spherical crystal shapes, such as the truncated octahedron. It should be stressed, however, that interfacial free energies only determine the equilibrium crystal shape and not the nonequilibrium shape that develops during growth. It is conceivable that the strongly octahedral crystal shape found in Ref. 47 is determined by kinetics.

In the previous section we found that the critical nucleus has mainly fcc character. Yet it still has considerably liquidlike and bcc-like character. In fact, it is not surprising that the critical nucleus has some liquidlike character. After all, it consists only of some 642 particles and has therefore a large surface-to-volume ratio. However, the bcc-like character is more intriguing. We have therefore studied the local order of the critical nucleus in more detail.

Given the spherical shape of the critical nucleus it is meaningful to calculate $f_{\text {liq }}, f_{\text {bcc }}$, and $f_{\text {fcc }}$ in a spherical shell of radius $r$ around the center-of-mass of the cluster. Figure 6 shows the radial profile of the local order of the critical nucleus. As expected, we find that the core of the nucleus is almost fully fcc-ordered and that far away from the center of the nucleus, $f_{\text {fcc }}$ decays to zero and $f_{\text {liq }}$ approaches unity. More surprisingly however, is that $f_{\text {bcc }}$ increases in the interface and becomes even larger than $f_{\text {fcc }}$, before it decays to zero in the liquid. Hence, the present simulations suggest that the fcc-like core of the equilibrated nucleus is "wetted" by a shell which has more bcc character. This finding explains why Fig. 4 shows that even fairly large nuclei do not have a pure fcc signature; there is always a residual bcc signature due to the interface. It also explains the strong bcc character of the small clusters, such as appear on the liquid side of the

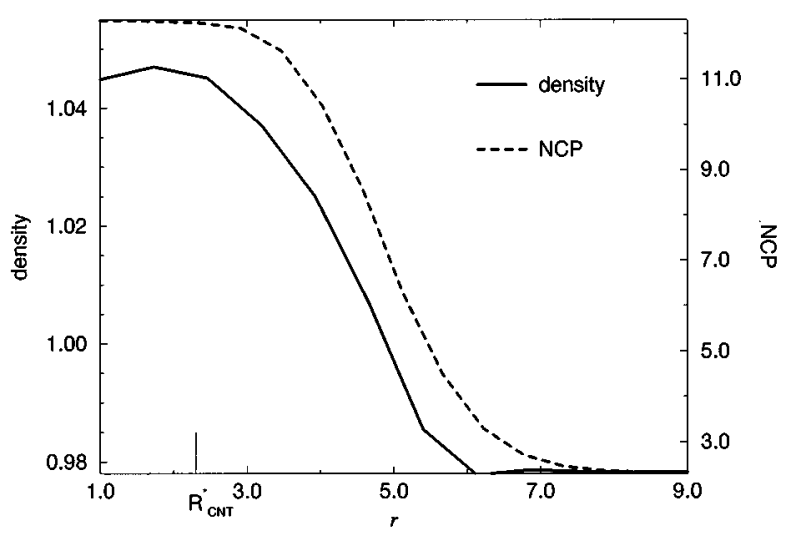

FIG. 7. The density and the number of connections per particle (NCP) as a function of $r$, the distance to the center-of-mass, for the critical nucleus in a Lennard-Jones system at $20 \%$ undercooling $(P=5.68, T=0.92)$. The coordinate-axes are such that they range from a liquid to a bulk solid value, both for the density and the structural order parameter. $R_{\mathrm{CNT}}$ is the radius of the critical nucleus as given by classical nucleation theory. Based on averages over 50 independent atomic configurations.

barrier; they are so small that their structure is strongly surface-dominated.

As can be seen from Fig. 6 the interface between the nucleus and the surrounding liquid is quite diffuse (some four atomic layers). Such a diffuse interface is predicted by recent theories of homogeneous nucleation. ${ }^{40,41}$ In contrast, classical nucleation theory assumes a sharp interface. A more specific prediction about the solid-liquid interface of crystal nuclei is made in the density functional theory of Harrowell and Oxtoby. ${ }^{40}$ This theory predicts that the density profile of the clusters reaches liquidlike values well before the orderparameter profile does. In other words, this theory predicts that there exists a "shell" with liquidlike density but solidlike order around the nucleus. To test this prediction we plotted both the density and the "degree of crystallinity" as measured by the number of bond-order connections per particle (NCP) (see Sec. III A). The number of such connections per particle is a measure for the local bond orientational order and can be used as a structural order parameter. Figure 7 shows the density and the number of connections per particle as a function of $r$. We see that the density in the core of the nucleus is somewhat lower than the density of the bulk fcc solid under similar conditions. In contrast, the structural order parameter reaches the same value in the core of the nucleus as in the bulk solid. This finding is in agreement with the density functional calculations of Ref. 40.

The figure also shows that both the density and the structural order parameter decay smoothly to a liquidlike value outside the nucleus. Moreover, as predicted theoretically, ${ }^{40}$ the density falls off faster than the structural order parameter. The latter profile appears to be displaced by some $0.7 \sigma$ with respect to the density profile. Hence the cluster is indeed surrounded by a thin layer that is liquidlike in density, but solidlike in structure. In fact, in the density-functional theory of Oxtoby ${ }^{49}$ the density change varies quadratically with the structural order parameter. Figure 8 shows the relation be- 


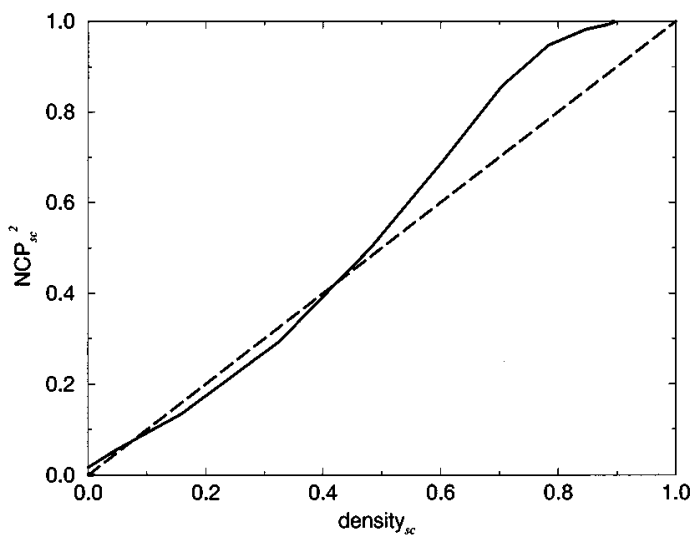

FIG. 8. Square of the scaled structural order parameter as a function of the scaled density for the critical nucleus in a Lennard-Jones system at $20 \%$ undercooling $(P=5.68, T=0.92)$. The scaled structural order parameter is given by $\mathrm{NCP}_{\mathrm{sc}} \equiv\left(\mathrm{NCP}-\mathrm{NCP}_{\text {liq }} / \mathrm{NCP}_{\text {sol }}-\mathrm{NCP}_{\text {liq }}\right)$, and the scaled density is given by density sc $_{\text {sc }} \equiv\left(\right.$ density - density $_{\text {liq }} /$ density $_{\text {sol }}-$ density $\left._{\text {liq }}\right)$, where NCP is the number of connections per particle, and liq and sol denote that the quantities are computed in the bulk liquid and bulk solid, respectively. The solid line is the result from the simulations, and the dashed straight line is the prediction of the density functional theory of Oxtoby (Ref. 49). Based on averages over 50 independent atomic configurations.

tween the square of the structural order parameter variation and the change in density, as obtained in the simulation. We should point out that our definition of the solid order parameter is not equivalent to the one used by Oxtoby. ${ }^{49}$ Still, the figure suggests that, at least far from the core (i.e., where the crystallinity and density is low) the quadratic relation between order parameter and density seems to be satisfied.

Both the diffuseness of the solid-liquid interface and the difference in the density and order-parameter profiles, make the definition of the size of the critical nucleus ambiguous. For instance, if we choose to locate the surface of the critical nucleus at the point where the order parameter is halfway between its bulk-solid and liquid values, then the radius of the nucleus would be $4.9 \sigma$ and the number of particles in the critical nucleus would be 630 . But if we use the halfway point of the density to define the crystallite surface, then we find a radius of $4.2 \sigma$, corresponding to 412 particles in the critical nucleus. A direct comparison of the size of the critical nucleus with the prediction of classical nucleation theory is therefore not very meaningful.

As the nucleus grows beyond its critical size, it retains its spherical shape and the core retains the same (fcc) crystal structure. More interestingly, the structure of the interface does not change either. The postcritical nuclei retain a high degree of bcc ordering at the interface and the density decays faster than the structural order parameter. In fact, as can be seen in Fig. 9, the width of the interface remains essentially constant. We have also studied the solid-liquid interface in the limit of an "infinitely large" crystal-nucleus, i.e., a planar interface. To this end, we brought the (100)-face of a slab of a thermally equilibrated fcc crystal in contact with a liquid and equilibrated the interface in a constant- $N V T$ molecular dynamics simulation $(\rho=0.978, \quad T=1.15, \quad N=$ 10532). Figure 10 shows that, just as with the small nuclei,

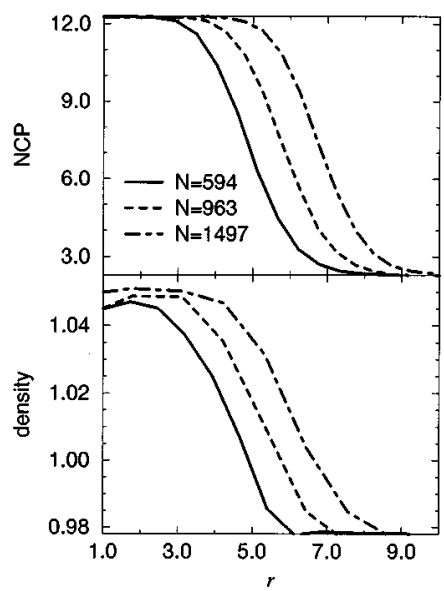

FIG. 9. The density and the number of connections per particle (NCP) as a function of $r$, the distance to the center-of-mass, for the critical nucleus and several postcritical nuclei in a Lennard-Jones system, at $20 \%$ undercooling $(P=5.68, T=0.92)$. Based on averages over 50 independent atomic configurations.

$f_{\text {fcc }}$ decreases monotonically in the interface while $f_{\text {bcc }}$ peaks there. The bcc-like structure of the fcc-liquid interface appears to be quite general and should be observable experimentally.

\section{Nucleation rate}

Up to this point, we have only discussed the static aspects of crystal nucleation. Let us now consider the actual barrier crossing process. Most of the previous computersimulation studies of nucleation rates were performed by rapidly quenching a liquid to temperatures well below its freezing point, and then measuring the time-lag until the first signs of crystallization appear. ${ }^{15,19-21,36,46,47}$ This method, although straightforward, has some disadvantages. First and foremost, as the nucleation rate depends exponentially on the degree of undercooling, the brute-force method only works

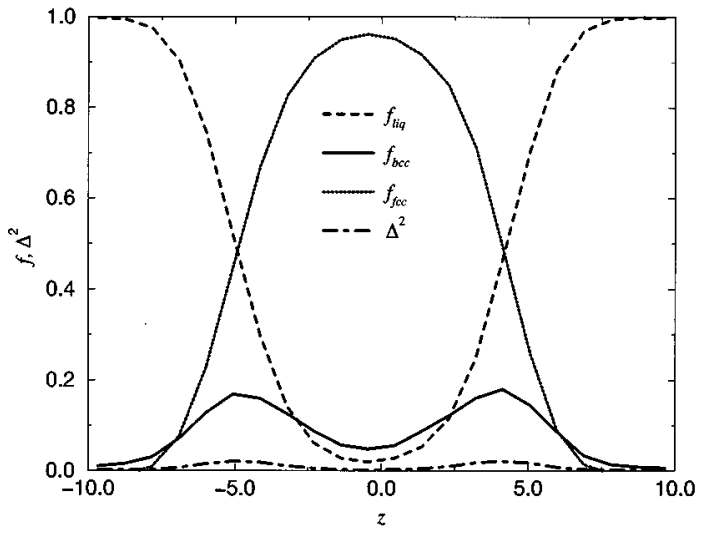

FIG. 10. The structural composition, indicated by $f_{\text {liq }}, f_{\text {bcc }}, f_{\text {fcc }}$, and $\Delta^{2}$, of the Lennard-Jones system with the planar fcc-liquid interface, equilibrated at coexistence $(\rho=0.978, T=1.15)$. The $z$-coordinate, in units of $\sigma$, is the coordinate perpendicular to the planar interface. Based on averages over 50 independent atomic configurations. 
under conditions of extreme supercooling. Moreover, the method lumps two times together. The first is the induction time, i.e., the time it takes the cluster-size distribution to respond to the temperature quench. In the stable liquid, only small clusters appear, whereas in the supercooled liquid there is an enhanced (although still very small) probability to observe larger clusters. The second is the actual time it takes to cross the nucleation barrier, given a (quasi) Boltzmann distribution of precritical nuclei. Finally, even in a strongly supercooled system, nucleation remains a rare event, and hence the statistics on the nucleation rate is usually poor.

We therefore did not use the "brute-force" approach to compute the nucleation rate. Rather, we employed the fact that nucleation is an activated process and that the rate is given by Eq. (6). The advantage of this approach is that we do not have to wait for the critical nucleus (or the activated state) to form spontaneously; we prepare the system at the top of the free energy barrier and simply measure the time correlation between the initial order-parameter velocity and the probability of finding the system in the solid side of the barrier at a later time $t$. This correlation function is expected to reach a plateau value relatively quickly (at least, compared to the actual times involved in the nucleation process) and hence the nucleation rate can be determined from comparatively short runs. By performing many runs, we can improve the statistical accuracy of the measurement of the nucleation rate. Even so, the simulations become quite time-consuming.

The first step in the computation of the flux is to identify the "transition state" from our knowledge of the shape of the free energy barrier. We denote this point by $Q_{6}^{*}$. We then performed a MD simulation of the system under the constraint $Q_{6}=Q_{6}^{*}$, to generate a set of independent configurations at the top of the barrier. Note that constraining $Q_{6}$ does not necessarily imply that the size of the critical nucleus is constrained. However, a structure analysis of the configurations at the top of the barrier showed that the average size of the largest cluster did not change significantly during the constrained run. The set of configurations obtained in this way was used as initial state for the computation of $R(t)$ from Eq. (10).

Figure 11 shows the transmission coefficient $\kappa$, as defined in Eq. (9) for $P=0.67$. The figure shows that initially the transmission coefficient decreases rapidly from the value $\kappa=1$ at $t=0$. This is due to recrossing at short times. However, after a short transient relaxation period of approximately $0.5 \tau, \kappa(t)$ appears to reach a plateau value (shown as a dashed line in the graph). As it is clear from the figure, the statistical accuracy of $\kappa(t)$ is rather poor, even though averages over 200 trajectories were taken. A direct analysis of the trajectories of the system in $Q_{6}$-space showed that its behavior is distinctly diffusive. The system does not clearly fall into either minima (solid or liquid one) in the duration of the run, but remains close to the top of the barrier in most cases. The largest cluster present in the system, the critical cluster, did not grow or shrink monotonically, but its size fluctuated, although in most cases a clear tendency to the liquid or to the solid minimum could be observed. Hence, to speak in the language of chemical kinetics, crystal nucleation

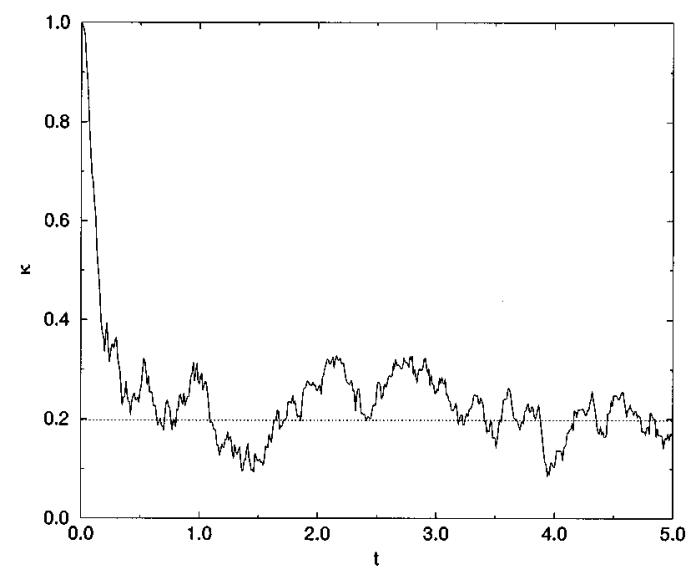

FIG. 11. Transmission coefficient as a function of time at $20 \%$ undercooling ( $P=0.67, T=0.6)$. The dotted line shows the plateau value that is established after $0.5 \tau$. This figure is based on averaging over 200 trajectories.

is closer to the Kramers limit of diffusive escape over a barrier ${ }^{50}$ than to the "ballistic" crossing of Eyring's transition-state theory. ${ }^{28}$

Indeed, due to the diffusive nature of the barrier crossing, the plateau value of the transmission coefficient is quite small, $\kappa \sim 0.05$ for $P=5.68$ and $\kappa \sim 0.2$ for $P=0.67$. The prediction of transition-state theory (TST) for the rate can be obtained by combining the initial value of the forward flux, $R\left(0^{+}\right)$, with the earlier results for the barrier height. It was found from the simulations that for $P=5.68$, $R\left(0^{+}\right)=5.85 \cdot 10^{-3} \tau^{-1}$, and hence $k_{\mathrm{TST}}=7.35 \cdot 10^{-14} \tau^{-1}$. From $k_{\text {TST }}$ and the plateau value of the transmission coefficient we can then get the full nucleation rate, which is found to be $k=4.04 \cdot 10^{-15} \tau^{-1}$. In the low pressure case the values were $k_{\mathrm{TST}}=2.40 \cdot 10^{-11} \tau^{-1}$ and $k=4.79 \cdot 10^{-12} \tau^{-1}$.

The rates obtained in our simulation are measured in units of $Q_{6}$ per unit time, as the quantity computed was the flux of $Q_{6}$. Nucleation rates are usually measured in number of solid particles produced in the unit volume per unit time. To get such a quantity for the results of the simulation we would have to multiply $k$ by $\rho_{\text {liq }} d N_{\text {sol }} / d Q_{6}$, where $\rho_{\text {liq }}$ is the density of the liquid and $N_{\text {sol }}$ is the number of solid particles. We assume that there is a linear relationship between $Q_{6}$ and the number of solid particles (this is certainly true for large crystallites). We then can write

$$
\frac{d N_{\mathrm{sol}}}{d Q_{6}}=\frac{N_{\mathrm{sol}}^{*}-N_{\mathrm{sol}}^{\mathrm{liq}}}{Q_{6}^{*}-Q_{6}^{\mathrm{liq}}}
$$

where the superscript liq denotes that the corresponding quantity is evaluated in the liquid minimum. Taking into account the results of the previous sections we finally obtain $k_{\mathrm{TST}}=2.23 \cdot 10^{-9} \sigma^{-3} \tau^{-1}, \quad k=1.23 \cdot 10^{-10} \sigma^{-3} \tau^{-1} \quad$ for $P=5.68$ and for $P=0.67$ we get $k_{\mathrm{TST}}=4.09 \cdot 10^{-7} \sigma^{-3} \tau^{-1}$, $k=8.19 \cdot 10^{-8} \sigma^{-3} \tau^{-1}$.

The value of the rate $k$ implies that in order to observe nucleation in a system of 10648 particles at $20 \%$ undercooling at $P=5.68$, a simulation time of the order of $10^{6} \tau$ would be required (this estimate is obtained by taking the inverse of 
$k$ and dividing the result by the volume of the system). Taking into account that a time step in a MD simulation is typically of the order of $10^{-2} \tau$, runs of a duration of at least $10^{8}$ time steps would have to be performed. This is not in disagreement with previous studies that were not able to see crystallization in liquids with a degree of undercooling smaller than about $26 \%$ during runs of a duration of $\sim 1000 \tau{ }^{47}$

\section{Comparison with classical nucleation theory}

Turnbull and Fisher ${ }^{51}$ applied the Becker-Döring formalism to nucleation in condensed systems and derived the following expression for the nucleation rate, ${ }^{1,51}$

$$
k=A(T) e^{-\Delta G^{*} / k_{B} T},
$$

where $\Delta G^{*}$ is the nucleation barrier and $A(T)$ is a kinetic prefactor. We are now in a position to test the predictions of classical nucleation theory, both concerning the height of the free-energy barrier to nucleation and the value of the kinetic prefactor. As argued in the previous section, predictions concerning the size of the critical nucleus are harder to test, as the size of the critical nucleus, as computed in the simulations, is ill defined.

In classical nucleation theory the height of the freeenergy barrier is given by ${ }^{1}$

$$
\Delta G^{*}=\frac{16 \pi \gamma^{3} v^{2}}{3(\Delta \mu)^{2}}
$$

where $\gamma$ is the surface free energy per unit area of the liquid-crystal interface, $v$ is the volume per particle in the solid, and $\Delta \mu$ is the difference in chemical potential between the bulk solid and bulk liquid.

Two problems arise when applying Eq. (20). The first is that we do not know the solid-liquid interfacial free energy for Lennard-Jones crystals in contact with a supercooled liquid. However, as already mentioned above, Broughton and Gilmer ${ }^{48}$ have calculated the surface free energy for three different orientations of the fcc crystal-liquid interface. They performed their calculations at coexistence, near the triple point (i.e., low pressure), and found the surface free energies to be equal within their error bars. In our comparison we will use the average of their estimates for the surface free energies of the different faces.

The second problem is that we do not know the difference in chemical potential between the bulk solid and bulk liquid at $20 \%$ undercooling. However, close to coexistence the difference in chemical potential can be approximated by ${ }^{1}$

$$
\Delta \mu \approx \Delta h\left(T_{m}-T\right) / T_{m},
$$

where $\Delta h$ is the enthalpy change per particle on freezing at coexistence and $T_{m}$ is the melting temperature. We have taken the enthalpy change per particle in the liquid-solid transition at coexistence from the data of Hansen and Verlet. ${ }^{42}$ In Table III we have collected for both pressures the average value of the surface free energies estimated by Broughton and Gilmer, ${ }^{48}$ the enthalpy change per particle on freezing at coexistence, ${ }^{42}$ the estimated difference in chemi-
TABLE III. Data used to calculate the nucleation rate as given by classical nucleation theory; the average of the surface free energies, $\gamma$, calculated by Broughton and Gilmer (Ref. 48), the enthalpy change per particle on freezing, $\Delta h$, at coexistence (Ref. 42), the estimated difference in chemical potential $\Delta \mu$ between the bulk fcc solid and bulk liquid at $20 \%$ undercooling, and the volume per particle in the bulk fcc solid at $20 \%$ undercooling, $v_{\text {fcc }}$, both for $P=0.67$ and $P=5.68$.

\begin{tabular}{ccccc}
\hline \hline$P$ & $\gamma$ & $\Delta h\left(T=T_{m}\right)$ & $\Delta \mu\left(T=0.8 T_{m}\right)$ & $v_{\mathrm{fcc}}\left(T=0.8 T_{m}\right)$ \\
\hline 0.67 & 0.35 & -1.31 & -0.262 & 0.998 \\
5.68 & 0.35 & -1.46 & -0.292 & 0.948 \\
\hline \hline
\end{tabular}

cal potential between the bulk fcc solid and bulk liquid at $20 \%$ undercooling and the volume per particle in the bulk fcc solid at $20 \%$ undercooling.

Using the data shown in Table III, classical nucleation theory yields the following predictions for the nucleation barriers: $G / k_{B} T=17.4$ at $P=0.67$ and $G / k_{B} T=8.2$ at $P=5.68$. We find from our simulations that $G / k_{B} T \approx 19.4$ for the lower pressure and $G / k_{B} T \approx 25.1$ for the higher pressure (see Fig. 3). As Broughton and Gilmer have calculated the surface free energy at a temperature and pressure which are closer to the temperature and pressure of the simulation at $P=0.67$, we expect the agreement between the theoretical prediction and the results of the simulation to be better for this lower pressure than for the higher pressure, $P=5.68$. In fact, for the lower pressure the agreement between the predicted height of the barrier and the height of the barrier as computed in our simulation is surprisingly good if one takes into account the crude approximations made in classical nucleation theory. The discrepancy for the higher pressure between the prediction of classical nucleation theory and the results of the simulation is most likely mainly due to the fact that the surface free energy at this higher pressure and temperature is somewhat larger than the Broughton and Gilmer estimate. As the surface free energy comes in with the third power in the theoretical expression for the height of the barrier, a difference of only $40 \%$ in the surface free energy could account for the discrepancy between theory and simulation. If we make the assumption that the surface free energy is proportional to the latent heat ${ }^{1}$ (which increases with pressure), then we arrive at an estimate for the barrier height at the higher pressure that is within $20 \%$ of the simulation results.

In classical nucleation theory the radius of the critical nucleus is given by ${ }^{1}$

$$
R^{*}=\frac{2 \gamma v}{|\Delta \mu|} \text {. }
$$

Using the data from Table III, classical nucleation theory gives the following predictions for the radius of a critical fcc nucleus: $2.7 \sigma$ for $P=0.67$ and $2.3 \sigma$ for $P=5.68$. We have indicated this radius for the critical nucleus at the higher pressure in Fig. 7. Although the exact boundary between the core and the interface of the nucleus is not clear, it seems that classical nucleation theory significantly underestimates the size of the critical nucleus. 
Let us next consider the kinetic prefactor. The following expression for $A(T)$ has been proposed ${ }^{1}$ :

$$
A(T)=Z \rho_{\mathrm{liq}} \frac{24 D n^{* 2 / 3}}{\lambda^{2}} .
$$

$D$ is the diffusion coefficient, $\rho_{\text {liq }}$ is the density of the liquid, $n^{*}$ is the size of the critical nucleus, and $\lambda$ is the atomic jump distance in the liquid. $Z$ is the Zeldovich factor, which relates the number of solid clusters in the steady state with the equilibrium value,

$$
Z=\left(\frac{\left|\Delta G^{\prime \prime}\left(n^{*}\right)\right|}{2 \pi k_{B} T}\right)^{1 / 2},
$$

where $\Delta G^{\prime \prime}\left(n^{*}\right)$ is the second derivative of the Gibbs free energy with respect to the cluster size at $n^{*}$. Using the expression of classical nucleation theory for $\Delta G$ we get

$$
Z=\left(\frac{|\Delta \mu|}{6 \pi k_{B} T n^{*}}\right)^{1 / 2} .
$$

When making the comparison between the classical nucleation theory prediction for $A(T)$ and the value obtained from the simulations, we use the value of $n^{*}$ obtained in the simulation. For $P=5.68$ we obtained in the simulation $n^{*} \sim 642$, so Eq. (25) leads to a value of the Zeldovich factor of $Z=5.12 \cdot 10^{-3}$, while for $P=0.67, n^{*} \sim 500$ and $Z=6.81 \cdot 10^{-3}$. Similar values are obtained if we use Eq. (24) directly, although in this case the statistical accuracy is poor. The appearance of the Zeldovich factor in the expression for the rate-constant is a consequence of the fact that the barrier crossing is considered as a diffusive rather than a ballistic process. ${ }^{1}$ It is precisely this diffusive behavior near the top of the barrier that leads to recrossings and hence to a reduction of $\kappa(t)$. The small value of the Zeldovich factor, as given by Eq. (24), is in qualitative agreement with the strong reduction of $\kappa$ due to recrossings, as found in the simulations.

The diffusion coefficient in the supercooled liquid was computed in a separate simulation and was found to be $D \approx 1 \cdot 10^{-2} \sigma^{2} \tau^{-1}$, for both pressures. The atomic jump distance was approximated by $\rho_{\text {liq }}^{-1 / 3}$, which gives $\lambda \approx 1.0 \sigma$ for $P=5.68$ and $\lambda \approx 0.97 \sigma$ for $P=0.67$. This leads to a prediction of the kinetic prefactor of $A=8.76 \cdot 10^{-2} \sigma^{-3} \tau^{-1}$ for $P=5.68$ and $A=0.113 \sigma^{-3} \tau^{-1}$ in the low pressure case. The value of the kinetic prefactor in the simulation can easily be obtained by dividing the value of the rate $k$ by $\exp \left(-\Delta G^{*} / k_{B} T\right)$. The resulting value is $A=9.78 \sigma^{-3} \tau^{-1}$ for $P=5.68$ and $A=21.83 \sigma^{-3} \tau^{-1}$ for $P=0.67$. This means that the kinetic prefactor obtained in the simulation is about two orders of magnitude larger than the one predicted by classical nucleation theory, leading to a larger value of the nucleation rate.

Broughton et al. ${ }^{52,53}$ performed a simulation study of crystal growth of a Lennard-Jones fcc crystal in contact with the melt. They observed that the (100) face crystallized two to three times faster than the (111) face. In fact, they found that for the (100) face the energy barrier for crystallization vanishes and that the rate is not limited by the mobility of atoms in the liquid, but is determined by the ideal gas thermal velocity, $\left(3 k_{B} T / m\right)^{1 / 2}$. If we assume that the growth mechanism of the critical nucleus is that of the (100) face, and take $A(T)$ to be

$$
A(T)=Z \rho_{\mathrm{liq}} \frac{4 n^{* 2 / 3}\left(3 k_{B} T / m\right)^{1 / 2}}{0.4 a},
$$

where $a$ is the interatomic spacing, ${ }^{52,53}$ then we get a predicted prefactor of $6.45 \sigma^{-3} \tau^{-1}$ for the higher pressure and $6.31 \sigma^{-3} \tau^{-1}$ for the lower pressure. Note that the agreement with the simulation results is much better, although the measured prefactors are still higher than the predicted ones. Most experiments also indicate that the kinetic prefactor is significantly larger than predicted by classical nucleation theory. ${ }^{1}$ However, it is interesting to note that in recent experiments by Brugmans et al. ${ }^{54}$ the opposite was found; a kinetic prefactor that is many orders of magnitude smaller than the estimate of classical nucleation theory.

Finally, we should point out that the nucleation rate at $20 \%$ supercooling, although very small on the time scale of a computer simulation, is still very large from an experimental point of view. If we use the values of argon for $\sigma$ and $\tau$, and express the nucleation rate at $P=5.68$ in the usual units, we find $k=1.44 \cdot 10^{24} \mathrm{~cm}^{-3} \mathrm{~s}^{-1}$. This means that liquid argon at $20 \%$ undercooling would crystallize essentially instantly. Indeed, argon cannot be supercooled by $20 \%$ (in fact, it is notoriously difficult to supercool liquid argon).

\section{ACKNOWLEDGMENTS}

This work was supported in part by "Scheikundig Onderzoek Nederland" (SON) with financial aid from NWO ("Nederlandse Organisatie voor Wetenschappelijk Onderzoek"). The work of the FOM Institute is part of the research program of "Stichting Fundamenteel Onderzoek der Materie" (FOM) and is supported by NWO. Part of the computation reported in this work was supported through NWO Grant No. MPR-94.2186. M.J. Ruiz-Montero acknowledges partial support from EU-HCM Grant No. ERBCHWICT941060 and Grant No. PB92-0683 from Dirección General de Investigación Científica y Técnica (Spain). We thank Peter Bladon and Willem Vos for a critical reading of the manuscript.

\section{APPENDIX A: ORDER PARAMETER DEFINITION}

First we define the set of neighbors of a particle $i$ as all particles $j$ that are within a given radius $r_{q}$ from $i$. The vectors $\mathbf{r}_{i j}$ joining neighbors are called bonds. The unit vector $\hat{\mathbf{r}}_{\mathbf{i j}}$ specifies the orientation of the bond $\mathbf{r}_{i j}$. In a given coordinate frame, the orientation of the unit vector $\hat{\mathbf{r}}_{\mathbf{i j}}$ uniquely determines the polar and azimuthal angles $\theta_{i j}$ and $\phi_{i j}$. In order to construct invariants, we first consider the spherical harmonics $Y_{l m}\left(\theta_{i j}, \phi_{i j}\right) \equiv Y_{l m}\left(\hat{\mathbf{r}}_{\mathbf{i j}}\right)$. We can now characterize the local structure around particle $i$ by

$$
\bar{q}_{l m}(i) \equiv \frac{1}{N_{b}(i)} \sum_{j=1}^{N_{b}(i)} Y_{l m}\left(\hat{\mathbf{r}}_{i j}\right),
$$


where the sum runs over all $N_{b}(i)$ bonds that particle $i$ has with its neighbors. The $\bar{q}_{l m}(i)$ are still local order parameters. By calculating the average of $\bar{q}_{l m}(i)$ over all $N$ particles, we obtain global orientational order parameters $\bar{Q}_{l m}$

$$
\bar{Q}_{l m} \equiv \frac{\sum_{i=1}^{N} N_{b}(i) \bar{q}_{l m}(i)}{\sum_{i=1}^{N} N_{b}(i)} .
$$

The $\bar{Q}_{l m}$ still depend on the choice of reference frame. However, from the $\bar{Q}_{l m}$, rotationally invariant combinations can be constructed,

$$
Q_{l} \equiv\left(\frac{4 \pi}{2 l+1} \sum_{m=-l}^{l}\left|\bar{Q}_{l m}\right|^{2}\right)^{1 / 2}
$$

and

$$
\widehat{W}_{l} \equiv W_{l} /\left(\sum_{m=-l}^{l}\left|\bar{Q}_{l m}\right|^{2}\right)^{3 / 2},
$$

with $W_{l}$ given by

$$
W_{l} \equiv \sum_{\substack{m_{1}, m_{2}, m_{3} \\
m_{1}+m_{2}+m_{3}=0}}\left(\begin{array}{ccc}
l & l & l \\
m_{1} & m_{2} & m_{3}
\end{array}\right) \bar{Q}_{l m_{1}} \bar{Q}_{l m_{2}} \bar{Q}_{l m_{3}} .
$$

$Q_{l}$ and $W_{l}$ are the second-order and third-order invariants, respectively. The term in parentheses in Eq. (A5) is a Wigner- $3 j$ symbol.

The order parameter $Q_{6}$ as defined above and used in Ref. 3 is not suited for constraint MD simulations, because the presence of a cutoff radius $r_{q}$ means that $Q_{6}$ is not a continuously differentiable function of all particle coordinates. This problem can be remedied by attributing a weight $\alpha\left(r_{i j}\right)$ to the contribution of a given pair $i j$ to the $Q_{l m}$, where $\alpha(r)$ is a function that goes to zero smoothly at $r=r_{q}$. In the present simulations, we have chosen $\alpha(r)$ to be a quadratic function that has its minimum at $r_{q}$ and equals one at $r_{i j}=\sigma$,

$$
\alpha\left(r_{i j}\right) \equiv\left(\frac{r_{i j}-r_{q}}{\sigma-r_{q}}\right)^{2} .
$$

The corresponding definitions of $\bar{q}_{l m}(i)$ is

$$
\bar{q}_{l m}(i) \equiv \frac{\sum_{j=1}^{N_{b}(i)} Y_{l m}\left(\hat{\mathbf{r}}_{i j}\right) \alpha\left(r_{i j}\right)}{\sum_{j=1}^{N_{b}(i)} \alpha\left(r_{i j}\right)},
$$

and $\bar{Q}_{l m}$ becomes

$$
\bar{Q}_{l m} \equiv \frac{\sum_{i=1}^{N} \sum_{j=1}^{N_{b}(i)} Y_{l m}\left(\hat{\mathbf{r}}_{i j}\right) \alpha\left(r_{i j}\right)}{\sum_{i=1}^{N} \Sigma_{j=1}^{N_{b}(i)} \alpha\left(r_{i j}\right)} .
$$

We find that the present definition of $Q_{6}$ leads to values that differ little from those obtained with the definition of $Q_{6}$ used in Ref. 3.

\section{APPENDIX B: LINKED LISTS FOR OCTAHEDRAL BOUNDARY CONDITIONS}

We use a truncated octahedral periodic unit cell in our simulations. At first sight, it would seem that a truncated

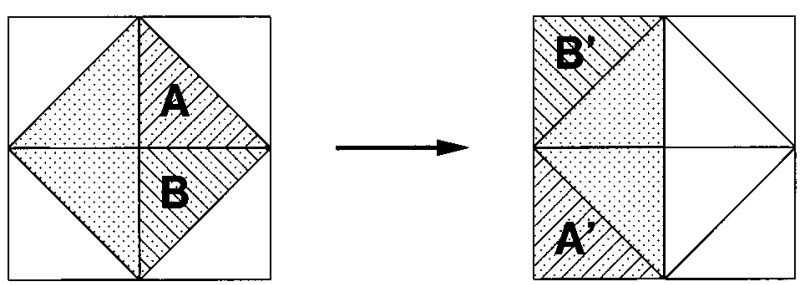

FIG. 12. The two-dimensional analog of the translation of all particles in the right half of the truncated octahedron, our simulation box, to the space between the left half of the truncated octahedron and the containing cube (containing the truncated octahedron), in order to set up the cell list. The rotated square in the middle corresponds to the truncated octahedron and the containing square corresponds to the containing cube. In this analog the translation of the particles corresponds to a translation of all particles in area $A$ to the area $A^{\prime}$ and all particles in area $B$ to the area $B^{\prime}$.

octahedron is not a convenient shape to use when setting up a mesh of cubic cells for the linked list that we use to speed up the computation. However, it should be noted that the truncated octahedron is a Wigner-Seitz cell of a bcc lattice. The unit cell of this cubic lattice has twice the volume of the truncated octahedron. Clearly, it is easy to partition this cubic unit cell into small cubic mesh cells. However, we should take care to avoid double counting, i.e., there should be a one-to-one correspondence between every point in the truncated octahedral box and one of the cubic mesh cells. To this end, we map all particles in the right half $(x>0)$ of the truncated octahedron to their periodic image that is in the left half $(x<0)$ of the cubic unit cell. This mapping is unique. And, as the volume of the truncated octahedron is equal to half the volume of the cube, we have thus mapped the position of every particle in our simulation box to the left half of the cube. Once we have mapped all particles to the left half of the cube, we can divide that volume into $0.5 M \times M \times M$ cells and use the conventional techniques to construct the linked list. ${ }^{30}$ Figure 12 shows a two-dimensional analog of this procedure. In the two-dimensional analog, all particles in area $A$ are mapped to area $A^{\prime}$ and all particles in area $B$ to area $B^{\prime}$.

${ }^{1}$ K. F. Kelton, in Crystal Nucleation in Liquids and Glasses, edited by $\mathrm{H}$. Ehrenreich and D. Turnbull (Academic, Boston, 1991), Vol. 45, pp. 75177.

${ }^{2}$ D. W. Oxtoby, J. Phys. Condensed. Matter 4, 7627 (1992).

${ }^{3}$ J. S. van Duijneveldt and D. Frenkel, J. Chem. Phys. 96, 4655 (1992).

${ }^{4}$ C. H. Bennett, in Algorithms for Chemical Computations, edited by R.E. Christofferson (American Chemical Society, Washington, D.C., 1977).

${ }^{5}$ D. Chandler, J. Chem. Phys, 68, 2959 (1978).

${ }^{6}$ E. A. Carter, G. Ciccotti, J. T. Hynes, and R. Kapral, Chem. Phys. Lett. 156, 472 (1989).

${ }^{7}$ G. Ciccotti, in Computer Simulations in Materials Science, edited by M. Meyer and V. Pontikis (Kluwer, Dordrecht, 1991), pp. 119-137.

${ }^{8}$ W. Ostwald, Z. Phys. Chem. 22, 289 (1897).

${ }^{9}$ I. N. Stranski and D. Totomanow, Z. Phys. Chem. 163, 399 (1933).

${ }^{10}$ S. Alexander and J. P. McTague, Phys. Rev. Lett. 41, 702 (1978).

${ }^{11}$ W. Klein and F. Leyvraz, Phys. Rev. Lett. 57, 2845 (1986).

${ }^{12}$ R. E. Cech, J. Met. 8, 585 (1956).

${ }^{13}$ H.-M. Lin, Y.-W. Kim, and T. F. Kelly, Acta Metall. 36, 2537 (1988).

${ }^{14}$ W. Löser, T. Volkmann, and D. M. Herlach, Mater. Sci. Eng. A178, 163 (1994).

${ }^{15}$ M. J. Mandell, J. P. McTague, and A. Rahman, J. Chem. Phys. 66, 3070 (1977). 
${ }^{16}$ M. Tanemura, Y. Hiwatari, H. Matsuda, T. Ogawa, N. Ogita, and A. Ueda, Prog. Theor. Phys. 58, 1079 (1977).

${ }^{17}$ J. N. Cape, J. L. Finney, and L. V. Woodcock, J. Chem. Phys. 75, 2366 (1981).

${ }^{18}$ M. J. Mandell, J. P. McTague, and A. Rahman, J. Chem. Phys. 64, 3699 (1976).

${ }^{19}$ C. S. Hsu and A. Rahman, J. Chem. Phys. 71, 4974 (1979).

${ }^{20}$ R. D. Mountain and A. C. Brown, J. Chem. Phys. 80, 2730 (1984).

${ }^{21}$ S. Nosé and F. Yonezawa, J. Chem. Phys. 84, 1803 (1986).

${ }^{22}$ J. Yang, H. Gould and W. Klein, Phys. Rev. Lett. 60, 2665 (1988).

${ }^{23}$ W. C. Swope and H. C. Andersen, Phys. Rev. B. 41, 7042 (1990).

${ }^{24}$ P. R. ten Wolde, M. J. Ruiz-Montero, and D. Frenkel, Phys. Rev. Lett. 75, 2714 (1995).

${ }^{25}$ L. D. Landau and E. M. Lifshitz, Statistical Physics, 3rd ed. (Pergamon, London, 1980).

${ }^{26}$ G. M. Torrie and J. P. Valleau, Chem. Phys. Lett. 28, 578 (1974).

${ }^{27}$ R. M. Lynden-Bell, J. S. van Duijneveldt, and D. Frenkel, Mol. Phys. 80, 801 (1993).

${ }^{28}$ S. Glasstone, K. J. Laidler, and H. Eyring, The Theory of Rate Processes (McGraw-Hill, New York, 1941).

${ }^{29}$ D. Chandler, Introduction to Modern Statistical Mechanics (Oxford University, New York, 1987).

${ }^{30}$ M. P. Allen and D. J. Tildesley, Computer Simulation of Liquids (Clarendon, Oxford, 1987).

${ }^{31}$ M. J. Ruiz-Montero and D. Frenkel (unpublished).

${ }^{32}$ P. J. Steinhardt, D. R. Nelson, and M. Ronchetti, Phys. Rev. B 28, 784 (1983).
${ }^{33}$ J. D. Honeycutt and H. C. Andersen, J. Phys. Chem. 90, 1585 (1986)

${ }^{34}$ A. D. J. Haymet, Chem. Phys. Lett. 107, 77 (1984).

${ }^{35}$ J. Yang, H. Gould, W. Klein, and R. D. Mountain, J. Chem. Phys. 93. 711 (1990).

${ }^{36}$ R. Eppenga and D. Frenkel, Mol. Phys. 52, 1303 (1984).

${ }^{37} \mathrm{~S}$. Nosé, in Computer Simulations in Materials Science, edited by M. Meyer and V. Pontikis (Kluwer, Dordrecht, 1991), pp. 21-41.

${ }^{38}$ D. J. Adams, CCP5 Q. 10, 30 (1983).

${ }^{39}$ A. M. Ferrenberg and R. H. Swendsen, Phys. Rev. Lett. 63, 1195 (1989).

${ }^{40}$ P. Harrowell and D. W. Oxtoby, J. Chem. Phys. 80, 1639 (1984).

${ }^{41}$ C. K. Bagdassarian and D. W. Oxtoby, J. Chem. Phys. 100, 2139 (1994).

${ }^{42}$ J. P. Hansen and L. Verlet, Phys. Rev. 184, 151 (1969).

${ }^{43}$ B. W. van de Waal, J. Chem. Phys. 90, 3407 (1989).

${ }^{44}$ J. Farges, M. F. de Feraudy, B. Raoult, and G. Torchet, J. Chem. Phys. 78, 5067 (1983)

${ }^{45}$ L. L. Boyer and J. Q. Broughton, Phys. Rev. B 42, 11461 (1990).

${ }^{46}$ J. D. Honeycutt and H. C. Andersen, J. Phys. Chem. 91, 4950 (1987).

${ }^{47}$ L. A. Báez and P. Clancy, J. Chem. Phys. 102, 8138 (1995).

${ }^{48}$ J. Q. Broughton and G. H. Gilmer, J. Chem. Phys. 84, 5759 (1986).

${ }^{49}$ D. W. Oxtoby (private communication).

${ }^{50}$ H. A. Kramers, Physica 7, 284 (1940).

${ }^{51}$ D. Turnbull and J. C. Fisher, J. Chem. Phys. 17, 71 (1949).

${ }^{52}$ J. Q. Broughton, G. H. Gilmer, and K. A. Jackson, Phys. Rev. Lett. 49, 1496 (1982).

${ }^{53}$ E. Burke, J. Q. Broughton, and G. H. Gilmer, J. Chem. Phys. 89, 1030 (1988)

${ }^{54}$ M. J. P. Brugmans and W. L. Vos, J. Chem. Phys. 103, 2661 (1995). 